\title{
On the Effect of Functionalizer Chain Length and Water Content in Polyethylene/Silica Nanocomposites: Part II - Charge Transport
}

\author{
M. Praeger, I. L. Hosier, A. F. Holt, A. S. Vaughan and S. G. Swingler \\ Electrical and Electronic Engineering Group, \\ University of Southampton, \\ Highfield, Southampton, \\ SO17 1BJ, U.K.
}

\begin{abstract}
The effects of functionalizer chain length and water content were explored in a series of polyethylene/silica nanocomposites. Silane molecules with differing chain lengths (propyl, octyl and octadecyl) were used to vary the nanoparticle surface chemistry, while vacuum drying and water immersion were used to extract water from or add water to samples previously equilibrated under ambient conditions. Electrical conductivity was found to be highly dependent upon water content, while space charge distributions measured using the pulsed electro-acoustic technique revealed that both the rate of charge injection at the electrode interfaces and the charge mobility within the sample bulk were strongly dependent on absorbed water. Changes to the charge transport dynamics due to the functionalizer chain length were, however, subtle. The removal of surface hydroxyl groups appears to be the primary mechanism by which functionalization influences electrical behavior; this reduces water uptake and, as a consequence, modifies charge transport behavior.
\end{abstract}

Index Terms - Conductivity, Nanocomposites, Polyethylene, Silica, Space Charge.

\section{INTRODUCTION}

THE utilization of nanocomposites for dielectric functions continues to attract attention, both in terms of academic research and in connection with the technological deployment of such systems in high voltage applications. However, while the potential benefits of this strategy are clear, there is ample evidence in the literature to demonstrate that the reproducible production of optimized systems with attractive combinations of properties is problematical.

Many researchers have already highlighted the crucial role that the nanoparticle/polymer interface [1-4] plays in determining the properties of nanodielectrics. Indeed, it has been argued that without the production of a modified interphase region through local nanoparticle/matrix interactions, it is difficult to account for the differing properties observed for micro- and nanocomposites of the same composition [5]. Surface functionalization has therefore been applied widely [3,6-10] in an attempt to manipulate nanoparticle/matrix interactions and consequent interphases. Typically, surface functionalization is considered in terms of its ability to reduce nanoparticle agglomeration in

Manuscript received on 22 December 2015, in final form XX Month 2016. order to achieve a homogeneous dispersion of particles throughout the matrix. Several possible mechanisms exist by which surface functionalization may act in this way, but in most cases, thermodynamic interactions or chemical bonding [11] between surface functional groups and the polymer matrix are assumed. It is also possible to completely encapsulate individual nanoparticles in a polymer brush [12], largely eliminating the possibility of subsequent agglomeration. Of course, functionalization also modifies the surface chemistry of the nanoparticles themselves, which leads to changes in the interactions that occur between the nanoparticles whilst in solution [13,14]. By applying two types of surface modification, some studies have even sought simultaneously to affect multiple processes $[15,16]$. In the work reported here, rather than seeking to manipulate nanoparticle dispersion, we focus on how modified nanoparticle surface chemistry impacts directly upon the electrical properties of the resulting nanodielectric.

A topic that has significant real-world consequences with respect to the technological deployment of nanocomposites concerns environmental effects, including the influence of exposure to water. Unfilled, non-polar polymers such as polyethylene are typically unaffected by storage under ambient 
conditions, whereas the extent of water absorption is significant in nanocomposites, even when samples are merely exposed to the environment. The location of absorbed water will then be determined both by interactions between the water and the nanoparticle surface and the solubility of the water in the matrix, such that a partition of the water between the bulk matrix and the nanoparticle surfaces occurs [17]. In nanodielectrics based upon non-polar polymers, absorbed water can accumulate preferentially at hydrophilic nanoparticle surfaces [4] and, consequently, surface functionalization can readily change the water absorption behavior of the system $[7,18,19]$. Indeed, in the accompanying publication [20], we suggest that absorbed water is a critical factor in the performance of nanodielectrics and that slight variations in this factor represent a potential source of much of the variability seen in the literature $[4,7,17,18]$.

In the case of breakdown strength, samples exposed to water always exhibit a reduced breakdown strength compared to dry equivalents $[7,19,20]$. When the breakdown strength of nanocomposites and the same unfilled polymer are compared, many examples can be found where the incorporation of the nanofiller (especially when non-fuctionalized) results in a loss of breakdown strength $[9,10,18,21]$. Conversely, other reports indicate no change in breakdown strength either under DC [2] or AC [22] conditions, whilst others indicate increased breakdown strength with surface treated nanosilica particles under both AC [19] and DC conditions [2,6]. Although variations in the quality of dispersion will exert some influence $[6,10,19,21-23]$, this cannot be the complete story, as evinced recently in the case of systems containing non-ideally dispersed calcined nanosilica [24].

Relatively few reports exist where electrical conduction is compared in nanosilica-based nanocomposites and the same unfilled polymer $[6,7,25,26]$. While three of these reports indicate an increase in electrical conductivity when the filler is added $[6,25,26]$, one indicates increased conductivity only at high filler loadings (12.5 wt \%) in samples that contain water, but unchanged conductivity both for dry samples and for samples with a lower (5 wt \%) filler loading [7].

In terms of space charge behavior, there are several reports of reduced space charge accumulation in nanocomposites when compared to micro-filled composites of the same formulation [27-29]. Other observations suggest that either heterocharge storage [7,19] or homocharge accumulation [10] may occur in nanofilled systems.

Previously, one of us employed silane chemistry to replace hydroxyl groups with propyl moieties on the surface of nanosilica and then examined the effect of this on the dielectric response of polyethylene/silica nanocomposites [8]. This showed that polar molecules, such as water, can be introduced in order to probe nanoparticle interfaces and demonstrated that changes in surface chemistry not only affect the uptake of water, but also the resulting aggregation state of the water. Subsequently, this probe technique was applied in an unfunctionalized polystyrene/nanosilica system [4] and, more recently, to demonstrate a change in surface chemistry via calcination in an HDPE/LDPE/nanosilica system [24].

The study reported here and in the preceding publication [20] considers a polyethylene/nanosilica system in which the surface chemistry of the nanofiller has been varied through the attachment of an approximately constant density of alkyl chains of varying length. In addition, specimens with each functionalizer chain length were pre-conditioned in order to vary the included water content. Specifically, the objective was to consider the relative importance of nanoparticle/matrix interactions and nanoparticle dispersion effects on electrical properties compared with consequential processes related to substituting hydroxyl with alkyl surface functionality. The latter is expected to have a dramatic effect on interfacial accumulation of water molecules and as a consequence to modify charge transport dynamics through the system. The accompanying paper considered water uptake kinetics, morphology, dielectric relaxation and breakdown; here, we report on conductivity and space charge accumulation.

\section{EXPERIMENTAL}

\subsection{MATERIALS AND SAMPLE PREPARATION}

Full details of the materials used, surface functionalization methods and sample preparation are provided in the accompanying paper [20] but, for convenience, the most important details are summarized below. The base polymer was a blend of $20 \%$ high density polyethylene (HDPE) (Rigidex HD5813EA; BP Chemicals) and $80 \%$ low density polyethylene (LDPE) (LD100BW; ExxonMobil Chemicals). The nanofiller was silicon dioxide $\left(\mathrm{SiO}_{2}\right)$ with a quoted

Table 1. Physical Structure and Data for the Three Functionalizing Agents Employed in this Study

\begin{tabular}{c|c|c|c|c}
\hline Functionalizer & Physical structure & Molar mass (g/mol) & Density (g/ml) & Amount used (ml) \\
\hline Trimethoxy(propyl)silane & $\mathrm{Si}-\left(\mathrm{OCH}_{3}\right)_{3}-\left(\mathrm{CH}_{2}\right)_{2}-\mathrm{CH}_{3}$ & 164.3 & 0.932 & 4.77 \\
Trimethoxyl(octyl)silane & $\mathrm{Si}-\left(\mathrm{OCH}_{3}\right)_{3}-\left(\mathrm{CH}_{2}\right)_{7}-\mathrm{CH}_{3}$ & 234.4 & 0.907 & 7.00 \\
Trimethoxyl(octadecyl)silane & $\mathrm{Si}-\left(\mathrm{OCH}_{3}\right)_{3}-\left(\mathrm{CH}_{3}\right)_{17}-\mathrm{CH}_{3}$ & 374.7 & 0.883 & 11.49 \\
\hline
\end{tabular}

Table 2. Blends used in these Investigations; Percentages are by Mass

\begin{tabular}{c|c|c}
\hline Blend & Base resin & Nano filler used \\
\hline CT1 & $20 \%$ HDPE, 80 \% LDPE & - \\
NS3 & $18 \%$ HDPE, 72 \% LPE & $10 \%$ nano Si functionalized with trimethoxy(propyl)silane \\
NS8 & $18 \%$ HDPE, 72 \% LDPE & $10 \%$ nano Si functionalized with trimethoxyl(octyl)silane \\
NS18 & $18 \%$ HDPE, 72\% LDPE & $10 \%$ nano Si functionalized with trimethoxyl(octadecyl)silane \\
\hline
\end{tabular}


particle size range of $10-20 \mathrm{~nm}$, in which the primary particles are present in a range of agglomerated structures [20]. Silane functionalizing agents with different alkyl tail lengths were attached to these particles [30] to give the systems specified in Tables 1 and 2. The amount of each silane used was in proportion to its molar mass, such that the number density of alkyl chains grafted to the nanosilica surface should be close to constant. The nanosilica and all silanes were obtained from Sigma Aldrich. All nanofilled samples contained $10 \mathrm{wt} \%$ of nanosilica and all samples were hot pressed into sheets $0.2 \mathrm{~mm}$ in thickness, crystallized isothermally at $115{ }^{\circ} \mathrm{C}$ for $1 \mathrm{~h}$ and then quenched in water [31]. This gives a morphology based upon a space-filling array of HDPE lamellae separated from one another by LDPE [32]. Previously, we showed that the inclusion of the nanosilica results in increased nucleation of the polymer and some disruption of lamellar growth [20].

To vary the water content, samples were subjected to one of three preconditioning regimes. Samples were immersed in water, dried in a vacuum oven or stored under ambient laboratory conditions for periods of up to $14 \mathrm{~d}$. In the case of the unfilled system, the water content was independent of preconditioning but, in the nanocomposites, the sample mass on vacuum drying decreased and asymptotically approached a constant value after $>7 \mathrm{~d}$, corresponding to the loss of between $0.37 \%$ and $0.53 \%$ of the initial mass, depending on the material formulation. On immersion in water, the mass of each sample increased, asymptotically approaching a value corresponding to an increase of between $0.70 \%$ and $0.82 \%$ relative to the initial sample mass over the same time scale.

\subsection{CHARACTERIZATION}

Samples for electrical conductivity measurements were sputter coated on both sides with $20 \mathrm{~mm}$ diameter gold electrodes and placed into a test cell containing opposing $20 \mathrm{~mm}$ electrodes. The testing system allows a constant DC voltage to be applied to the specimen and the resulting current measured. Two test programs were used: a ramped voltage test, where the voltage was increased in $100 \mathrm{~V}$ steps with a $10 \mathrm{~s}$ delay between applying the voltage and measuring the current; a constant voltage test, where the voltage was held constant and the current measured every $60 \mathrm{~s}$ for $3 \mathrm{~h}$. The applied voltage was limited to $8 \mathrm{kV}(40 \mathrm{kV} / \mathrm{mm})$ to prevent breakdown.

Space charge measurements were performed using a $5 \mathrm{Lab}$ "Peanuts" pulsed electro-acoustic (PEA) system. A DC poling voltage of up to $8 \mathrm{kV}$ was applied to the sample using a semiconductive anode electrode, whilst a $600 \mathrm{~V}, 50$ ns pulse was used to obtain measurements. Charging measurements were performed for up to $1 \mathrm{~h}$, after which, decay measurements were performed with the specimen short circuited. All measurements were conducted under ambient conditions and the time delay between sample removal from the preconditioning environment and commencement of the test was minimized.

\section{RESULTS AND DISCUSSION}

\subsection{VOLTAGE DEPENDENCE OF ELECTRICAL CONDUCTIVITY}

Figure 1 shows conductivity results obtained using the ramped voltage protocol. From Figure 1a, it is evident that the conductivity of the unfilled polymer matrix initially decreases with increasing field $(0-10 \mathrm{kV} / \mathrm{mm})$ before progressively increasing. In an experiment of this type, the measured current, and hence the derived conductivity, depend upon two factors for any given sample geometry. First, due to polarization effects, the current will decay with time after the initial application of the electric field and asymptotically approach the true conduction current. Second, when the applied field is increased, in general, the conductivity will also increase. Thus, if the residence time at each step is very long, the latter term dominates while, if it is short, the behavior will reflect both factors. The data shown in Figure 1 are therefore a consequence of the relatively short hold time at each applied voltage - an expedient employed to enable data to be acquired at many different applied voltages in a reasonable time. Comparing the different unfilled samples (CT1), it is apparent that all exhibit comparable behavior; pre-conditioning in

(a)

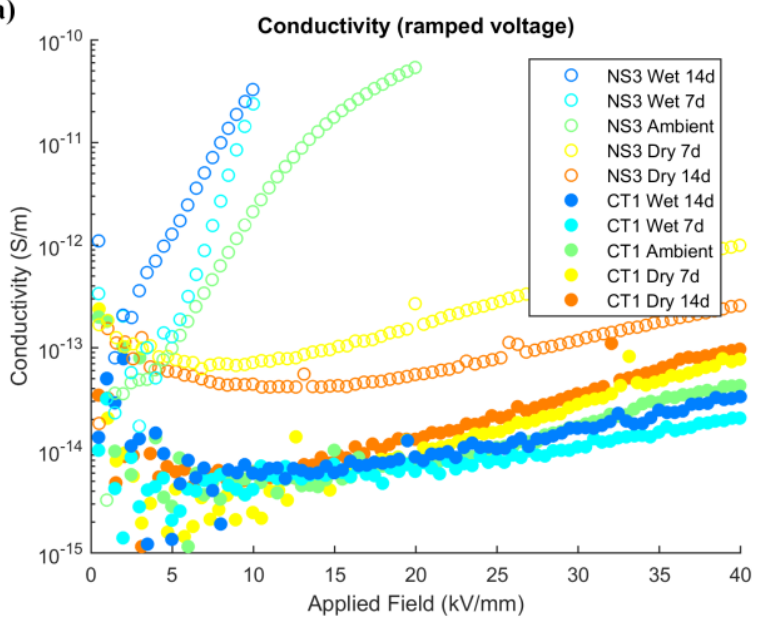

(b)

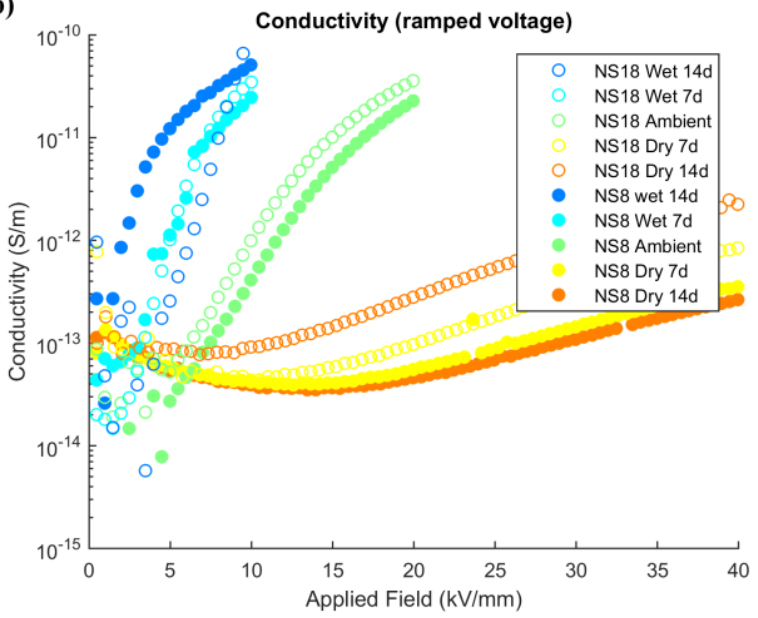

Figure 1. Electrical conductivity from stepped voltage experiments. (a) Data obtained from CT1 (closed symbols) and NS3 (open symbols). (b) Data obtained from NS8 (closed symbols) and NS18 (open symbols). 
vacuum or in water has little effect on the conductivity at any given voltage. For all CT1 systems, the conductivity varies with applied field within the range $4 \times 10^{-15} \mathrm{~S} / \mathrm{m}$ (close to the minimum measureable conductivity) to $\sim 5 \times 10^{-14} \mathrm{~S} / \mathrm{m}$.

The behavior of all nanocomposites is, however, markedly different. First, all exhibit increased conductivity values relative to the unfilled reference. Second, in the case of both the ambient samples and those examined after immersion in water, the conductivity is much more strongly dependent upon the applied field than in CT1. Third, at all fields above the initial range where polarization effects dominate, the conductivity is observed to increase dramatically with water content. For example, considering the data obtained at $10 \mathrm{kV} / \mathrm{mm}$, the conductivity increases progressively in the sequence CT1, NS3 (Dry 14 d), NS3 (Dry 7 d), NS3 (ambient), NS3 (Wet $7 \mathrm{~d}$ ) and NS3 (Wet $14 \mathrm{~d}$ ) from $\sim 5 \times 10^{-15}$ $\mathrm{S} / \mathrm{m}$ to nearly $10^{-10} \mathrm{~S} / \mathrm{m}$. The behavior of ambient samples is closer to that of wet samples, indicating that in studies where samples have not been subjected to prolonged drying, moisture absorbed from the ambient environment will have a significant effect on DC conduction and other electrical properties. However, comparison of the systems prepared with the differently functionalized nanosilicas suggests that this factor is far less influential than water content.

(a)

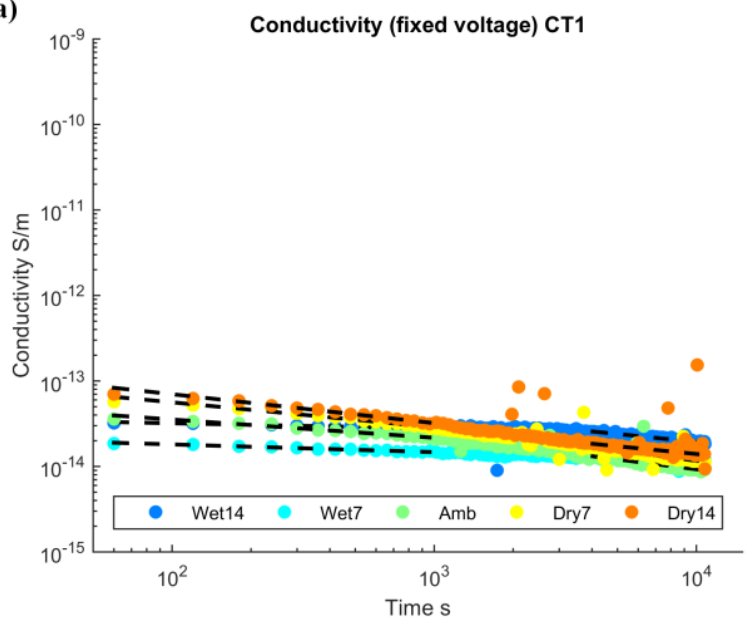

(c)

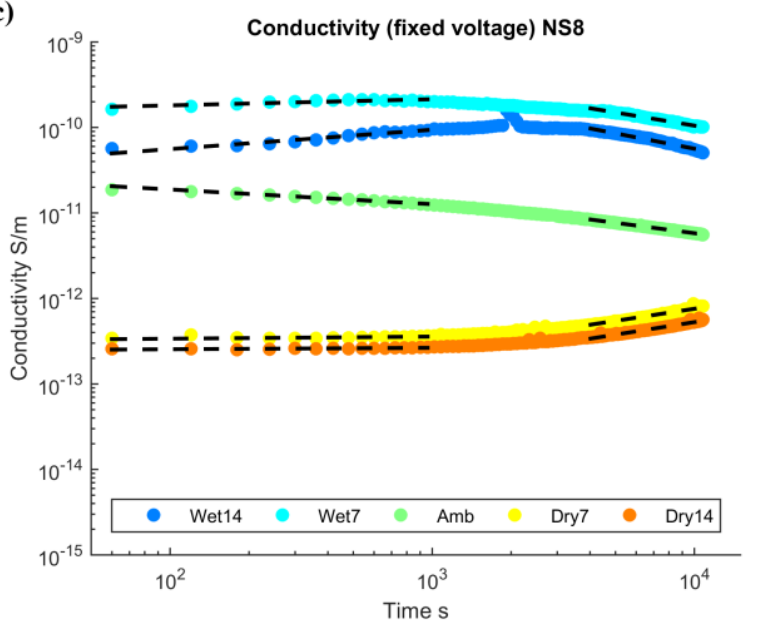

\subsection{TIME DEPENDENCE OF ELECTRICAL CONDUCTIVITY}

The form of the conductivity versus applied field plots shown in Figure 1 is strongly influenced by polarization effects and, consequently, the variation of conductivity with time was examined. However, in view of the wide range of conductivities evident in Figure 1, all measurements could not be conducted using the same applied voltage. Rather, in order to restrict the conductivity to measureable values $\left(<10^{-9} \mathrm{~S} / \mathrm{m}\right)$ and to avoid any risk of electrical breakdown, which could damage the equipment, testing of the nanocomposites was conducted at $10 \mathrm{kV} / \mathrm{mm}, 20 \mathrm{kV} / \mathrm{mm}$ and $40 \mathrm{kV} / \mathrm{mm}$ for waterimmersed, ambient and dried samples respectively. CT1 was also tested at $40 \mathrm{kV} / \mathrm{mm}$. The resulting constant voltage conductivity/time plots are shown in Figure 2.

According to the literature [33], the variation of the absorption current with time often follows a power law relationship (Curie-von Schweidler Law):

$$
I=A t^{-b_{n}}
$$

where $I$ is the current, $t$ is the time after the application or removal of the external field, $A$ is a temperature dependent factor and $b_{n}$ is a constant representing the slope of the log

(b)

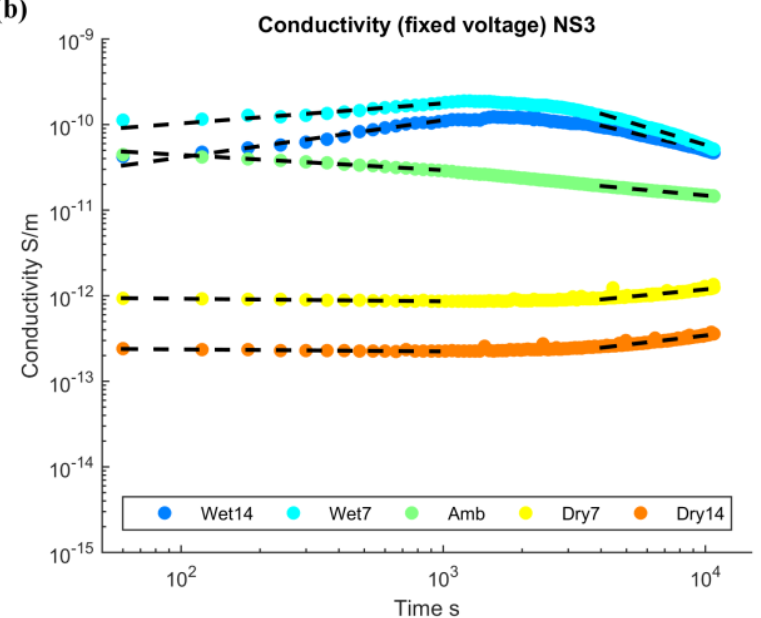

(d)

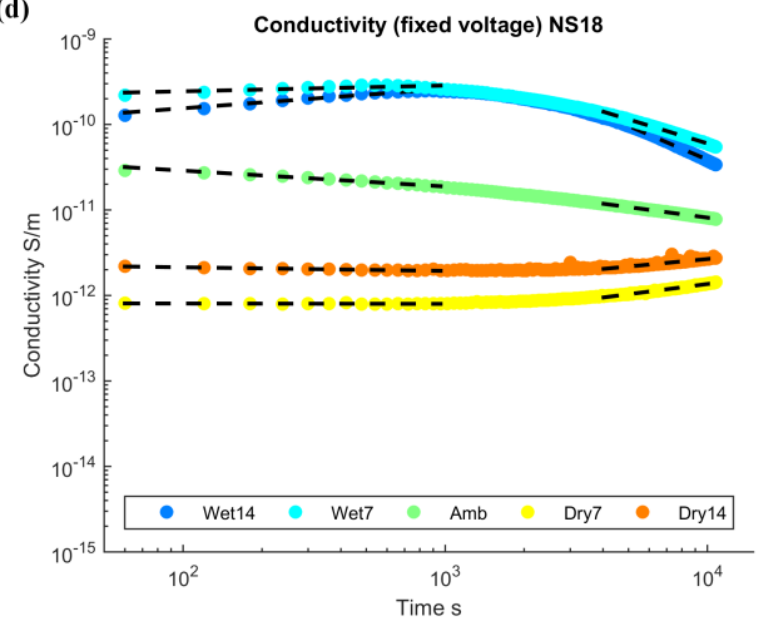

Figure 2. Electrical conductivity from constant voltage experiments. (a) Data obtained from CT1. (b) Data obtained from NS3. (c) Data obtained from NS8. (d) Data obtained from NS18. Dashed lines show the fits to equation (1) in the short and long time ranges. 
Table 3. $b_{n}$ Parameters Obtained by Fitting the Conductivity/Time Data to Equation (1)

\begin{tabular}{|c|c|c|c|c|c|c|c|c|}
\hline \multirow{2}{*}{$\begin{array}{c}\text { Sample } \\
\text { Condition }\end{array}$} & \multicolumn{2}{|c|}{ CT1 } & \multicolumn{2}{|c|}{ NS3 } & \multicolumn{2}{|c|}{ NS8 } & \multicolumn{2}{|c|}{ NS18 } \\
\hline & Regime $1\left(b_{I}\right)$ & Regime $2\left(b_{2}\right)$ & Regime $1\left(b_{1}\right)$ & Regime $2\left(b_{2}\right)$ & Regime $1\left(b_{1}\right)$ & Regime $2\left(b_{2}\right)$ & Regime $1\left(b_{1}\right)$ & Regime $2\left(b_{2}\right)$ \\
\hline Wet 14 & 0.061 & 0.309 & -0.434 & 0.714 & -0.227 & 0.596 & -0.228 & 1.308 \\
\hline Wet 7 & 0.090 & 0.083 & -0.235 & 0.913 & -0.072 & 0.511 & -0.067 & 0.935 \\
\hline Ambient & 0.214 & 0.383 & 0.178 & 0.279 & 0.172 & 0.404 & 0.186 & 0.409 \\
\hline Dry 7 & 0.291 & 0.405 & 0.030 & -0.295 & -0.025 & -0.538 & 0.005 & -0.397 \\
\hline Dry 14 & 0.338 & 0.302 & 0.023 & -0.360 & -0.016 & -0.486 & 0.042 & -0.292 \\
\hline
\end{tabular}

current/log time plot. However, in analyzing the current/time data obtained here, these results were separated into two time ranges; short-term behavior from 0 to $1000 \mathrm{~s}$ (regime 1, characterized by $b_{1}$ ) and long-term data from 4000 to $10800 \mathrm{~s}$ (regime 2, characterized by $b_{2}$ ). The fits based on equation 1 were made over both time ranges and are shown in Figure 2 (dashed lines); derived $b_{n}$ parameter values are shown in Table 3. In the case of the unfilled CT1 (Figure 2a), the response is independent of pre-conditioning and for all these samples, the data are close to linear when plotted on log-log axes. The gradient (and hence $b_{n}$ ) remains comparable over the short-time and long-time regimes, indicating that the conductivity varies with time in accordance with equation 1 throughout the experiment. Also, for the unfilled polymer, exposure to water has only a minor effect on the electrical conductivity, which is close to the sensitivity limit of our apparatus; the measured conductivity is comparable across the complete conditioning range, from $14 \mathrm{~d}$ in vacuum to $14 \mathrm{~d}$ immersion in water.

Similar time dependent behavior was exhibited by all of the nanocomposites that were allowed to equilibrate with their environment (i.e. the green points labelled Amb in Figure 2). For these ambient conditioned samples, the gradient of the log conductivity/log time plot again remains relatively constant, indicating that the behavior of these samples also conforms to equation 1.

Consider now the data obtained from the six nanocomposites that had been dried for either $7 \mathrm{~d}$ or $14 \mathrm{~d}$ (yellow and orange data points). For such systems, there is a significant change in behavior between regime 1 and regime 2, corresponding to an increase in conduction current with time (this causes $b_{n}$ to decrease sharply, becoming negative in regime 2). As such, these samples do not conform to the standard behavior indicated by equation 1; a similar time dependent variation in conductivity has, however, been reported elsewhere for nanocomposite samples that had been thoroughly dried prior to testing [26].

Finally, the six samples that had previously been immersed in water exhibit yet another form of behavior, in which the conductivity initially increases over the first $2000 \mathrm{~s}$ of the experiment, before progressively falling. This behavior is reflected in a significant increase of $b_{n}$ in regime 2 (see Table 3).

The data shown in Figures 1 and 2 demonstrate that the absolute value of the conductivity of all nanocomposites is markedly influenced by their water content. In the case of the ambient specimens, they are in equilibrium with their surroundings and, for these systems, no significant variations in water content would be expected during the $3 \mathrm{~h}$ duration of the experiments conducted here. Consequently, in the absence of any other time-varying factors, the dependence of conductivity on time is of the conventional form, as given by equation 1. In the case of the samples pre-conditioned in vacuum, their water content is reduced significantly compared with the ambient value and, therefore, it is reasonable to assume that, during the course of an experiment conducted under ambient laboratory conditions, their water content will increase somewhat and the conductivity with it. In the case of specimens previously immersed in water the converse argument applies.

While this direct association of the time variations in overall water content leading to time variations in electrical conductivity is qualitatively appealing, it is not consistent with the water absorption/desorption kinetics reported previously [20], where significant variations in sample mass only occur over considerably longer timescales. The conclusion must therefore be that a different mechanism with a less direct relationship to water adsorption/desorption applies.

\subsection{SPACE CHARGE}

An extensive range of space charge measurements was made to support the conductivity data presented above. For each combination of sample type and preconditioning regime, both charge accumulation and charge decay were measured as a function of time, leading to 40 independent time evolutions. Only a small subset of these data can be shown here.

1) Charge Accumulation: Very little space charge is accumulated in the unfilled samples, regardless of sample preconditioning and, consequently, no data are included here. In contrast, all nanosilica-filled samples display significantly increased levels of charge storage $[7,10,19]$ and, furthermore, the exhibited behavior is strongly dependent on sample preconditioning. When taken as a whole, the most striking feature of these space charge data is the transition from heterocharge accumulation in wet nanocomposite samples to homocharge accumulation in vacuum dried ones, as illustrated for NS8 in Figure 3.

Figure 4 shows representative time dependent space charge data for the NS3 system after (a) preconditioning in water for $14 \mathrm{~d}$ and (b) after vacuum drying for $14 \mathrm{~d}$; the behavior shown is shared by all of the nanofilled samples. From Figure 4a, it is evident that heterocharge gradually accumulates near both the anode and cathode; an additional subtlety is that after about $600 \mathrm{~s}$, the heterocharge that has accumulated near the cathode progressively decreases, which results in a concurrent decrease in the amount of charge on this electrode. During the timescale 


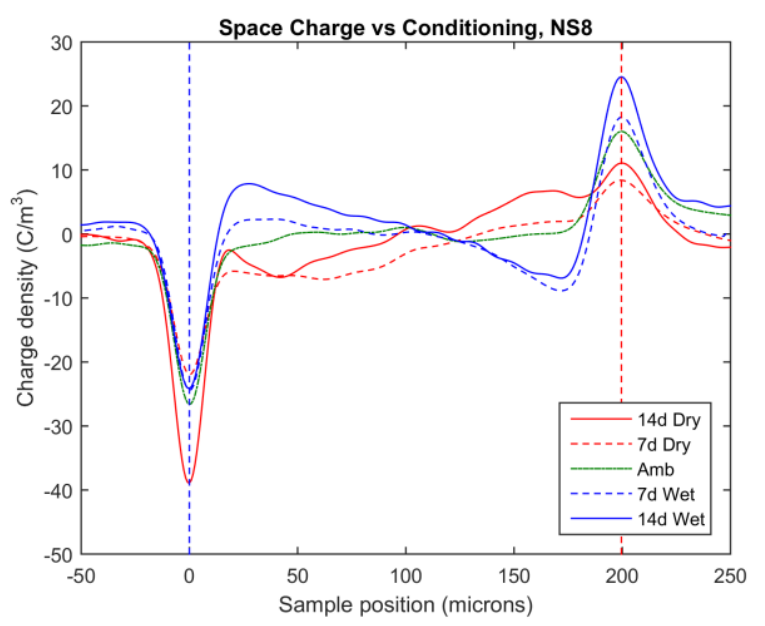

Figure 3. Space charge data for NS8, charged at $25 \mathrm{kV} / \mathrm{mm}$ for $3600 \mathrm{~s}$, where the curves illustrate the transition from heterocharge for waterimmersed samples to homocharge for vacuum dried ones.

of these experiments no equivalent variation of heterocharge at the anode occurred.

For the vacuum-dried sample, homocharge is observed within the bulk of the material $[9,10]$, as illustrated in Figure $4 \mathrm{~b}$. Packets of homocharge are formed and appear slowly to migrate away from the electrodes into the bulk of the material. Between the electrode charge at the sample surface and the homocharge packets, regions of reduced homocharge develop. Indeed, after $3600 \mathrm{~s}$, some heterocharge is evident immediately adjacent to the cathode. No equivalent process occurs at the anode.

Similar space charge profiles to those described above have been explained through the interplay between two processes, which occur simultaneously [19]. Heterocharge production was attributed to auto-ionization of water, whereupon, $\mathrm{OH}^{-}$and $\mathrm{H}_{3} \mathrm{O}^{+}$ions are formed within the bulk of the material. This will occur in regions of high electric stress where water is present (i.e. near to nanoparticle surfaces), which explains the absence of this effect in the unfilled samples. These ions then migrate towards the electrodes under the applied field, being captured

(a)

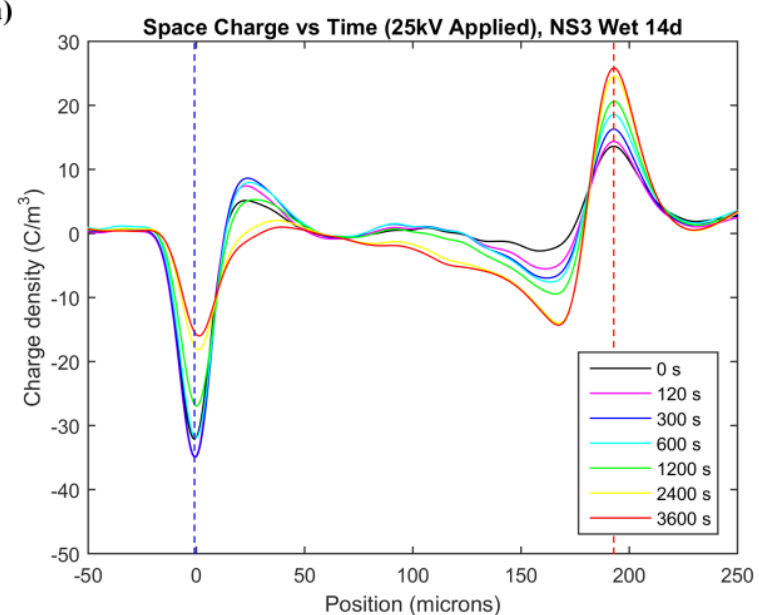

by local traps that exist within nanosilica/polymer interface layers $[2,7,9,19]$. Simultaneously, a high electric field at the electrodes leads to Schottky injection of homocharge in the form of electrons and holes. The PEA system measures net charge, such that the observed space charge profile represents the superposition of heterocharge from water ionization, homocharge from carrier injection and the electrode charge.

The space charge behavior of nanocomposite samples is evidently strongly affected by the presence of water. Although the exact nature of the ionized water is somewhat contentious [34], the neutralization of charge observed at the cathode implies that recombination of positive ions with injected electrons (at the cathode) is more likely, or occurs more quickly, than the equivalent recombination of negative ions with holes near to the anode. The time delay between heterocharge accumulation and neutralization may be due to the mobility of the positive ions $\left(\mathrm{H}_{3} \mathrm{O}^{+}\right.$for example), which is expected to be low compared to that of injected electrons. Also, as heterocharge accumulates, the local electric field at the sample surface will increase, which should cause the rate of charge injection to increase.

For samples that had been vacuum dried prior to space charge examination, it is likely that the same two mechanisms of space charge creation are present, but in the opposite ratio. That is, homocharge creation by injection at the electrodes initially dominates and as the sample charging time increases, more and more homocharge is created. This progressively advances into the bulk of the material, but does so slowly because of the low electrical conductivity of the system. The dip in homocharge that develops near to the electrodes may be related to two factors: reduced homocharge injection or superposition of heterocharge from ionization of water that is diffusing into the sample from its environment. That is, during the course of each space charge measurement, the sample will tend to equilibrate with the surrounding environment, which will predominantly modify the water content at the sample surface, until the entire sample reaches equilibrium. Charge injection is likely to be critically dependent on the conditions

(b)

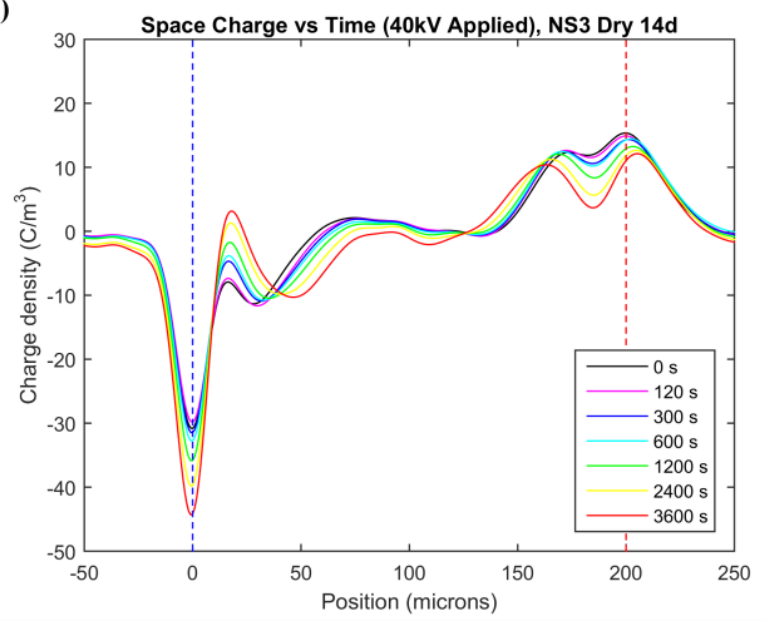

Figure 4. Space charge data obtained from (a) NS3 preconditioned in water for $14 \mathrm{~d}$ and then charged at $25 \mathrm{kV} / \mathrm{mm} \mathrm{for} 3600 \mathrm{~s}$ and (b) from NS3 dried in vacuum for $14 \mathrm{~d}$ and then charged at $40 \mathrm{kV} / \mathrm{mm}$ for $3600 \mathrm{~s}$. The curves illustrate a general the transition from heterocharge in water-immersed samples to homocharge in vacuum dried ones. 
at the electrode-polymer interface and, consequently, although a change in the local water content adjacent to the electrodes would equate to only a small change in the total water content/sample mass, this could nevertheless have a dramatic effect on the rate of charge injection.

(a)

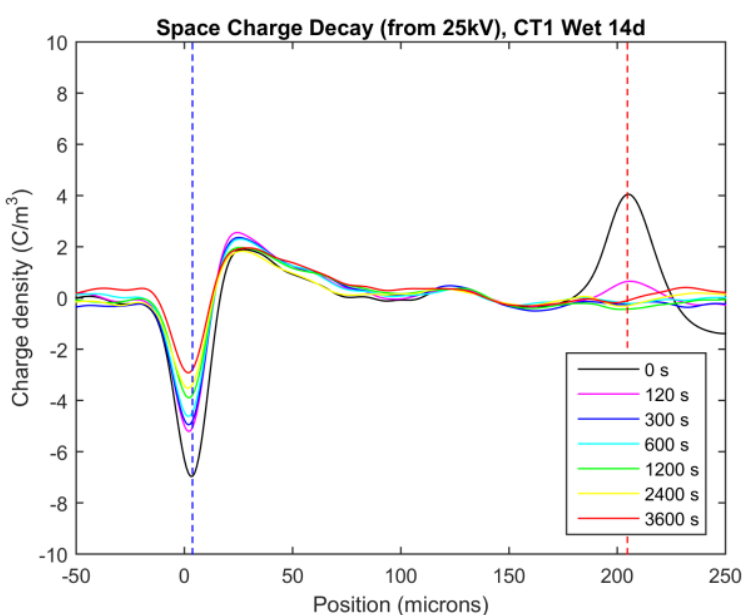

(b)

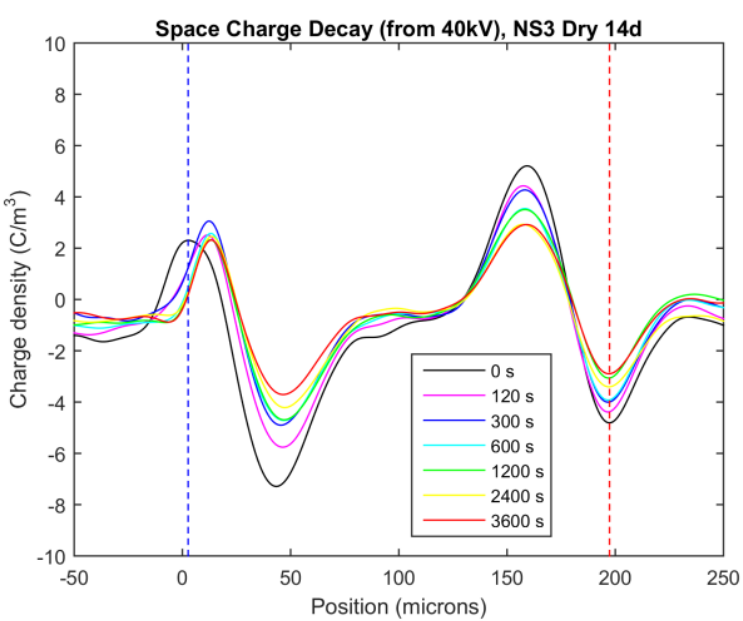

(c)

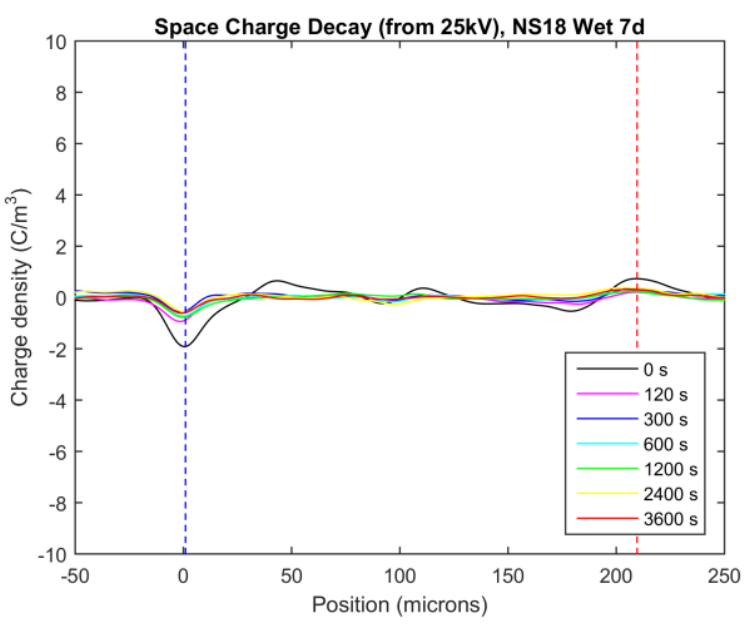

Figure 5. Representative space charge decay data. Samples charged for 1 hour prior to short circuiting, times indicate the delay between short circuiting and measurement of space charge. (a) Unfilled sample CT1 after $14 \mathrm{~d}$ immersed in water; sample charged at $25 \mathrm{kV} / \mathrm{mm}$. (b) Sample NS3 after $14 \mathrm{~d}$ of drying; sample charged at $40 \mathrm{kV} / \mathrm{mm}$. (c) Sample NS18 after $7 \mathrm{~d}$ immersed in water; sample charged at $25 \mathrm{kV} / \mathrm{mm}$.
2) Charge Decay: Charge decay after the externally applied voltage was removed and the sample short circuited was measured for each combination of sample type and preconditioning regime. In the discussion that follows it is convenient to continue to refer to the electrodes as the anode and cathode, despite the fact that there is no externally applied voltage. Similarly, homocharge and heterocharge take their meanings from the polarity of the externally applied voltage, prior to its removal. Once again, the nanocomposite samples display broadly equivalent behavior regardless of functionalizer chain length and, consequently, only a representative subset of the data is shown. The unfilled CT1 samples are again markedly different in character to the nanocomposites, being relatively invariant with preconditioning. Figure 5a shows representative data obtained from CT1 after immersion in water for $14 \mathrm{~d}$, which reveals a small quantity of residual heterocharge near the cathode, which decays only slightly over the time period considered.

Figures $5 \mathrm{~b}$ and $5 \mathrm{c}$ contain complementary data that show the discharge behavior of the vacuum dried and water immersed nanocomposite samples respectively. From Figure 5b, it is evident that, in dried samples, the measurements are dominated by a large quantity of homocharge stored inside the sample with heterocharge induced at both electrodes. Conversely, for all nanocomposites pre-conditioned under ambient or wet conditions, the amount of stored charge that could be detected during discharging was found to be minimal. Where a measurable quantity of stored charge was seen, this was present as heterocharge just inside the material, which dissipated completely in less than $120 \mathrm{~s}$ (see Figure 5c). It was therefore not possible to reliably evaluate the relative effect of the different functionalizers, due to inherent experimental limitations. Rapid charge dissipation leading to low charge storage is consistent with the relatively high conductivity of these water-containing samples, while the presence of heterocharge is consistent with the space charge observed whilst the external voltage was applied and with the suggestion of auto-ionized water as the charge carriers.

\section{CONCLUSION}

The behavior of polyethylene nanocomposites containing 10 wt $\%$ of nanosilica, functionalized with molecules of three different alkyl chain lengths, has been compared with an unfilled reference blend. The rationale is that by varying the length of the attached alkyl chains, interactions between the nanofiller and the matrix polymer will vary. However, structural characterization of the resulting nanocomposites indicates that while the nanosilica increases the nucleation density and perturbs the lamellar habit of the polymer, these effects are not significantly altered by changes to the nanoparticle surface chemistry. The inclusion of nanosilica does, however, have a dramatic effect on water absorption, whereby water accumulates at nanoparticle/polymer interfaces and the DC breakdown strength is dramatically reduced [20].

In the case of the unfilled polymer blend, electrical conductivity was found to be largely independent of 
preconditioning treatment (vacuum drying, equilibration with the laboratory environment or immersion in water); negligible absorption of water occurs in the unfilled system. Conversely, in all three nanocomposites, the magnitude of the conductivity was found to be strongly dependent upon preconditioning treatment; that is, on water content. In addition, examination of the time dependence of conductivity demonstrates that, for samples that are not in equilibrium with their environment, anomalous variations of conductivity occur with time. While it is tempting to ascribe this to variations in the bulk water content of the system, quantitative comparison of the above timescale (hundreds of seconds) with the duration over which water absorption occurs (days to weeks) indicates that this is not so. Rather, examination of space charge data suggests that change in surface condition as a result of diffusion of water into or out of the specimen affects the rate of charge injection at the electrode-sample interface and that this plays a major role in determining the observed charge transport.

The differences between the nanocomposites formulated with the differently functionalized nanosilicas were in general small compared to experimental errors and it was not possible to discern any consistent dependence of electrical properties upon the silane alkyl chain length. If any such dependence exists, it was dwarfed by the large-scale changes imposed by variations in water content. Consequently, we conclude that the primary mechanism for the marked variations in breakdown behavior, dielectric response, conductivity and space charge behavior seen in this study is variation in water content which, in turn, affects charge transport dynamics within the system. At least within the systems studied here, it would therefore seem that the primary mechanism by which functionalization of the nanosilica surfaces with alkyl chains affects macroscopic electrical properties is not through changed interactions between the nanofiller and the matrix (variations in nanoparticle dispersion, changed interphase structures, etc.) but, rather, through consumption of hydroxyl groups, which directly reduces water absorption and indirectly affects charge transport dynamics.

\section{ACKNOWLEDGMENT}

The authors gratefully acknowledge the RCUK's Energy Program for the financial support of this work through the Top \& Tail Transformation program grant, EP/I031707/1 (http://www.topandtail.org.uk/).

\section{REFERENCES}

[1] T. J. Lewis, "Nanometric dielectrics," IEEE Transactions on Dielectrics and Electrical Insulation Vol. 1, pp. 812-825, 1994.

[2] T. Tanaka, "Dielectric nanocomposites with insulating properties," IEEE Transactions on Dielectrics and Electrical Insulation, Vol. 12, pp. 914-928, 2005.

[3] M. Roy, J. K. Nelson, R. K. MacCrone, L. S. Schadler, C. W. Reed, R. Keefe, and W. Zenger, "Polymer nanocomposite dielectrics - The role of the interface," IEEE Transactions on Dielectrics and Electrical Insulation, Vol. 12, pp. 629-643, 2005.

[4] M. Praeger, A. S. Vaughan, and S. G. Swingler, "A dielectric spectroscopy study of the polystyrene/nanosilica model system," IEEE
International Conference on Solid Dielectrics (ICSD), pp. 859-862, 2013.

[5] M. Praeger, T. Andritsch, S. G. Swingler, and A. S. Vaughan, "A simple theoretical model for the bulk properties of nanocomposite materials," IEEE Conference on Electrical Insulation and Dielectric Phenomena (CEIDP), 699-702, 2014.

[6] M. Roy, J. K. Nelson, R. K. MacCrone, and L. S. Schadler, "Candidate mechanisms controlling the electrical characteristics of silica/XLPE nanodielectrics," Journal of Materials Science, Vol. 42, pp. 3789-3799, 2007.

[7] L. Hui, J. K. Nelson, and L. S. Schadler, "The influence of moisture on the electrical performance of XLPE/silica nanocomposites," IEEE International Conference on Solid Dielectrics (ICSD), 2010.

[8] K. Y. Lau, A. S. Vaughan, G. Chen, I. L. Hosier, and A. F. Holt, "On the dielectric response of silica-based polyethylene nanocomposites," Journal of Physics D-Applied Physics, Vol. 46, 2013.

[9] K. Y. Lau, A. S. Vaughan, G. Chen, I. L. Hosier, and A. F. Holt, "On nanosilica surface functionalization using different aliphatic chain length silane coupling agents," IEEE International Conference on Solid Dielectrics (ICSD), pp. 896-899, 2013.

[10] K. Y. Lau, A. S. Vaughan, G. Chen, I. L. Hosier, A. F. Holt, and K. Y. Ching, "On the space charge and DC breakdown behavior of polyethylene/silica nanocomposites," IEEE Transactions on Dielectrics and Electrical Insulation, Vol. 21, pp. 340-351, 2014.

[11] J. Zhu, H. Q. Peng, F. Rodriguez-Macias, J. L. Margrave, V. N. Khabashesku, A. M. Imam, K. Lozano, and E. V. Barrera, "Reinforcing epoxy polymer composites through covalent integration of functionalized nanotubes," Advanced Functional Materials, Vol. 14, pp. 643-648, 2004.

[12] X. F. Ding, J. Z. Zhao, Y. H. Liu, H. B. Zhang, and Z. C. Wang, "Silica nanoparticles encapsulated by polystyrene via surface grafting and in situ emulsion polymerization," Materials Letters, Vol. 58, pp. 31263130, 2004.

[13] R. P. Bagwe, L. R. Hilliard, and W.H. Tan, "Surface modification of silica nanoparticles to reduce aggregation and nonspecific binding," Langmuir, Vol. 22, pp. 4357-4362, 2006.

[14] L. Vaisman, G. Marom, and H. D. Wagner, "Dispersions of surfacemodified carbon nanotubes in water-soluble and water-insoluble polymers," Advanced Functional Materials, Vol. 16, 357-363, 2006.

[15] S. Virtanen, T. M. Krentz, J. K. Nelson, L. S. Schadler, M. Bell, B. Benicewicz, H. Hillborg, and S. Zhao, "Dielectric breakdown strength of epoxy bimodal-polymer-brush-grafted core functionalized silica nanocomposites," IEEE Transactions on Dielectrics and Electrical Insulation, Vol. 21, pp. 563-570, 2014

[16] W. Yani, Z. Jian, and Z. Hanying, "Functional colloidal particles stabilized by layered silicate with hydrophilic face and hydrophobic polymer brushes," Journal of Polymer Science, Part A: Polymer Chemistry, Vol. 47, pp. 15351543, 2009.

[17] A. Sami, M. F. Frechette, E. David, and S. Savoie, "Water as a digression relative to the dielectric response in the frequency domain for polymer composites," Annual Report Conference on Electrical Insulation and Dielectric Phenomena, pp. 501-504, 2008.

[18] I. L. Hosier, M. Praeger, A. F. Holt, A. S. Vaughan, and S. G. Swingler, "Effect of water absorption on dielectric properties of nanosilica/polyethylene composites," IEEE Conference on Electrical Insulation and Dielectric Phenomena (CEIDP), pp. 651-654, 2014.

[19] L. Hui, L. S. Schadler, and J. K. Nelson, "The Influence of Moisture on the Electrical Properties of Crosslinked Polyethylene/Silica Nanocomposites," IEEE Transactions on Dielectrics and Electrical Insulation, Vol. 20, pp. 641-653, 2013.

[20] I. L. Hosier, M. Praeger, A. F. Holt, A. S. Vaughan, and S. G. Swingler, "On the effect of functionaliser chain length and water content in polyethylene/nano-silica composites: Part I - Electrical Breakdown and Dielectric Spectroscopy," Submitted for publication, IEEE Transactions on Dielectrics and Electrical Insulation, 2015.

[21] M. Praeger, A. S. Vaughan, and S. G. Swingler, "The breakdown strength and localised structure of polystyrene as a function of nanosilica fill-fraction," IEEE International Conference on Solid Dielectrics (ICSD), pp. 863-866, 2013.

[22] G. Iyer, R. S. Gorur, R. Richert, A. Krivda, and L. E. Schmidt, "Dielectric properties of epoxy based nanocomposites for high voltage 
insulation," IEEE Transactions on Dielectrics and Electrical Insulation, Vol. 18, pp. 659-666, 2011.

[23] I. L. Hosier, M. Praeger, A. S. Vaughan, and S. G. Swingler, "Electrical properties of polymer nano-composites based on oxide and nitride fillers," IEEE Electrical Insulation Conference (EIC), pp. 438-441, 2015.

[24] M. Praeger, I. L. Hosier, A. S. Vaughan, and S. G. Swingler, "The effects of surface hydroxyl groups in polyethylene-silica nanocomposites," IEEE Electrical Insulation Conference (EIC), pp. 201204, 2015 .

[25] S. L. Abd-El Messieh and N. N. Rozik, "Dielectric and morphological studies on polyester/nanosilica fume composites," Journal of Applied Polymer Science, Vol. 122, pp. 714-721, 2011.

[26] K. Y. Lau, A. S. Vaughan, G. Chen, I. L. Hosier, and A. F. Holt, "Absorption current behaviour of polyethylene/silica nanocomposites," Dielectrics 2013, Journal of Physics Conference Series, Vol. 472, 2013.

[27] J. K. Nelson, J. C. Fothergill, L. A. Dissado, and W. Peasgood, "Towards an understanding of nanometric dielectrics," IEEE Conference on Electrical Insulation and Dielectric Phenomena (CEIDP), pp. 295298, 2002.

[28] R. C. Smith, C. Liang, M. Landry, J. K. Nelson, and L. S. Schadler, "The mechanisms leading to the useful electrical properties of polymer nanodielectrics," IEEE Transactions on Dielectrics and Electrical Insulation, Vol. 15, pp. 187-196, 2008.

[29] J. Castellon, H. N. Nguyen, S. Agnel, A. Toureille, M. Frechette, S. Savoie, A. Krivda, and L. E. Schmidt, "Electrical properties analysis of micro and nano composite epoxy resin materials," IEEE Transactions on Dielectrics and Electrical Insulation, Vol. 18, pp. 651-658, 2011.

[30] L. J. Brown, R. C. D. Brown, and R. Raja, "Heterogenisation of ketone catalysts within mesoporous supports for asymmetric epoxidation," RSC Advances, Vol. 3, pp. 843-850, 2013.

[31] I. L. Hosier, A. S. Vaughan, and S. G. Swingler, "An investigation of the potential of ethylene vinyl acetate/polyethylene blends for use in recyclable high voltage cable insulation systems," Journal of Materials Science, Vol. 45, pp. 2747-2759, 2010.

[32] I. L. Hosier, A. S. Vaughan, and S. G. Swingler, "Structure-property relationships in polyethylene blends: the effect of morphology on electrical breakdown strength," Journal of Materials Science, Vol. 32, pp. 4523-4531, 1997.

[33] T. C. Guo and W. W. Guo, "A transient-state theory of dielectricrelaxation and the Curie-VonSchweidler law," Journal of Physics CSolid State Physics, Vol. 16, pp. 1955-1960, 1983.

[34] D. Marx, M. E. Tuckerman, J. Hutter, and M. Parrinello, "The nature of the hydrated excess proton in water," Nature, Vol. 397, pp. 601-604, 1999.

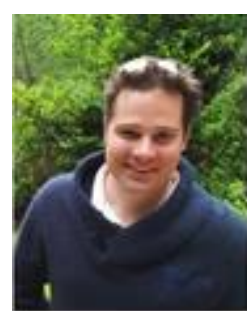

Matthew Praeger has an M.Phys degree in Physics with Laser Science and received a Ph.D for Laser Generation of X-rays, both from the University of Southampton. He subsequently worked at the Optoelectronics Research Centre before moving to the Electrical and Electronic Engineering group where his research has included electrical phenomena such as dielectrophoresis and nanocomposite materials for dielectric applications.

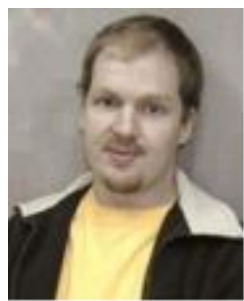

Ian Hosier was born in the UK in 1970 . He received the B.Sc. degree in physics and the Ph.D. degree in polymer physics from The University of Reading, UK. He then went on with postdoctoral research at the same university, which involved extensive collaborations with institutions in Germany and Japan. In 2001 he moved to Florida State University in the USA before returning to the UK to take a research post at the University of Southampton.

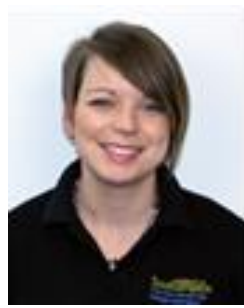

Alex Holt graduated from the University of Southampton with M.Chem. and Ph.D degrees in chemistry. After completing her PhD, she went on to works on a multidisciplinary project involving corrosion of copper and silver in transformers which utilizes a range of analytical techniques.

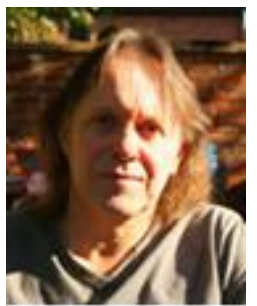

Alun Vaughan (SM'07) has a B.Sc. degree in chemical physics and a Ph.D. degree in polymer physics. After working at the UK's Central Electricity Research Laboratories and spending a period as an academic at The University of Reading, he is now Professor of Dielectric Materials and Head of the Electronics and Electrical Engineering research group at the University of Southampton. He is a former chair of The Dielectrics Group of the Institute of Physics, a Fellow of the Institute of Physics and a Fellow of the IET.

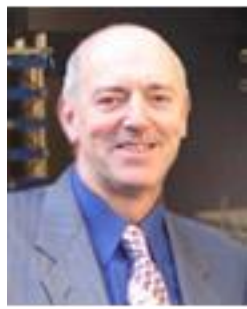

Steve Swingler received the B.A. and Ph.D. degrees in physics from the University of Lancaster. In 1976 he was appointed Pirelli Postdoctoral Research Fellow at Southampton University, investigating dielectrics for superconducting and extruded dc cables. He joined the Central Electricity Research Laboratories at Leatherhead in 1979 working mainly on novel cable systems and insulating materials. In 1990 he transferred to the National Grid Company, becoming Cables Manager and then Company Undergrounding Adviser. In 2003 he joined the School of Electronics \& Computer Science at Southampton University as Professor in Transmission Asset Engineering. He is the UK Member of CIGRE Study Committee B1 (High Voltage Cables) 